\title{
A LISP program to determine similarity relations in letter displays
}

\author{
IAN MORRISON \\ Queen's University, Kingston, Ontario K7L 3N6, Canada
}

\begin{abstract}
Similarity relations between letters are important in displays used in tachistoscopic recognition experiments. A LISP program is described that calculates similarity relations on the basis of an empirical letter-similarity matrix or on the basis of feature sharing in a feature-set definition supplied by the user. With either derivation method, there are three options for the type of average to be calculated for target display: (1) the average for the whole target, (2) the average for each letter in the target paired to all other letters in the target, and (3) the average of each letter in the target paired with its immodiate neighbors.
\end{abstract}

In theoretical models of letter recognition and text comprehension, similarity between characters is a useful concept. Of the many ways to define similarity, two definitions are utilized here: (1) Similarity is represented by the degree of confusion between letters in a confusion matrix (e.g., Gilmore, Hersh, Caramazza, \& Griffin, 1979), and (2) similarity is the proportion of features shared by two letters compared to the total number of features defining both letters (e.g., Briggs \& Hovecar, 1975). The first definition derives similarity empirically, whereas the second determines similarity computationally. Both methods are useful for researchers investigating, or controlling for, the effects of similarity in letter recognition experiments or in computer simulations of theoretical models. The program described below is intended as an aid to researchers considering similarity relations. Use of the program allows the user to equate word or pseudoword targets for similarity at the level of the whole target or of individual letters using either a user-specified feature set or an empirically derived similarity matrix. The program has been tested by applying not only the feature sets produced by Geyer and Dewald (1973) and by Keren and Baggen (1981), but also the empirical matrix produced by Gilmore et al. (1979) to pseudowords that varied in approximation to English (Hirata \& Bryden, 1971).

The program was implemented in RT-11 LISP for the PDP-11 computer (RT-11 LISP was written by Jeffrey Kodosky, 1977, and is available through the DECUS librarian). There is no particular reason for using a PDP-11 (in fact, the program was originally written on

Computing facilities were provided by Grant A-9581 to B. E. Butler from the Natural Sciences and Engineering Research Council. Reprint requests should be directed to Ian Morrison, Department of Psychology, Queen's University, Kingston, Canada. a VAX-11/750 in FRANZ LISP), and the program could be altered to run on a different machine.

There are reasons for using LISP. First, LISP is a symbol-manipulating language, a concept familiar to all cognitive psychologists. If letters or their internal representations can be considered to be symbols, we should use a language specifically designed to manipulate symbols. Second, LISP is an interactive language that can alter its environment, leaving information in a form accessible by subsequent programs. For example, programs that generate knowledge bases for use in letter recognition can leave an enriched environment for programs that simulate that process. Third, LISP is the most common language in the artificial intelligence field and, also, the most frequently used by cognitive psychologists implementing their theoretical models. These reasons are only a few of the arguments in favor of LISP mentioned by Winston and Horn (1981).

A similarity value exists for each pair of letters. The program calculates average similarity values in one of three ways. First, the average of the similarity values for all possible pairs of letters in a target can be computed. Second, the average can be calculated for each letter in the target paired with all others in the target. Third, the average can be calculated for each letter paired with its immediate neighbors. The similarity values can be calculated using the empirical or the computational derivation.

Examples of the required input to the program and its output are shown below. For either derivation method, a list of targets is required as input. The list is simply a file of words or pseudowords. If the empirical derivation is selected, the program will request the file name for a list of empirically derived similarity values (in percentage form) for each possible letter comparison. The file is in list form and can be envisioned as a list of lists, each of which is a row representing the upper diagonal half of a confusion matrix. The general format, 
using an example of the similarity matrix produced by Gilmore et al. (1979), is of the form:

$$
\begin{array}{ll}
{\left[\left(\mathrm{a} \mathrm{s}_{\mathrm{a}} \mathrm{s}_{\mathrm{b}} \ldots \mathrm{s}_{\mathrm{z}}\right)\right.} & {[(\mathrm{a} 100102 \ldots 9402)} \\
\left(\mathrm{b} \mathrm{s}_{\mathrm{b}} \mathrm{s}_{\mathrm{c}} \ldots \mathrm{s}_{\mathrm{z}}\right) & (\mathrm{b} 100423 \ldots 5323) \\
\ldots & \ldots \\
\left.\left(\mathrm{z} \mathrm{s}_{\mathrm{z}}\right)\right] & (\mathrm{z} 100)]
\end{array}
$$

If the computational method is selected, the program will request the file name for a definition of the alphabet using a particular feature set. The general format of the input, using an example with mnemonics for the feature set produced by Geyer and Dewald (1973), is shown below. (The letter "T" is replaced by the "*" character, since " $\mathrm{T}$ " has special significance to the LISP system. The replacement is not necessary for the file of words, but it is required for the file of empirical percentage values as well.) Feature definition input should be of the form:

$$
\begin{array}{cl}
{\left[\left(a\left(f_{1} f_{2} \ldots f_{a}\right)\right)\right.} & {[(a \text { (esp esm oii oii obh osv }))} \\
\left(b\left(f_{1} f_{2} \ldots f_{b}\right)\right) & (b(\text { ev ecs ecs owh oii osh })) \\
\ldots & \ldots \\
\left.\left(z\left(f_{1} f_{2} \ldots f_{z}\right)\right)\right] & (z \text { (eh eh owh owh }))]
\end{array}
$$

The similarity relations can be derived by empirical or computational means. The goal of the program is to calculate the average for a whole target, the average for each letter in a target, or the average for each letter paired with its immediate neighbors. What is the algorithm? Table 1 shows an empirical similarity matrix and a feature definition for each letter in a hypothetical character set. To calculate the average for the whole display, $\mathrm{ABCD}$, we would obtain the similarity values for all pairs: $A B, A C, A D, B C, B D$, and $C D$. From the empirical table, these values are $67 \%, 0 \%, 20 \%, 40 \%$, $33 \%$, and $25 \%$, respectively. The average for the whole

\begin{tabular}{|c|c|c|c|c|}
\hline & A & B & C & $\mathrm{D}$ \\
\hline $\begin{array}{l}\text { A } \\
\text { B } \\
\text { C } \\
\text { D }\end{array}$ & 100 & $\begin{array}{r}67 \\
100\end{array}$ & $\begin{array}{r}0 \\
40 \\
100\end{array}$ & $\begin{array}{r}20 \\
33 \\
25 \\
100\end{array}$ \\
\hline \multicolumn{5}{|c|}{$\begin{array}{l}{\left[\left(A\left(F_{0} F_{1} F_{2} F_{3} F_{6}\right)\right)\right.} \\
\left(B\left(F_{0} F_{2} F_{3} F_{4} F_{5} F_{6} F_{7}\right)\right) \\
\left(C\left(F_{3} F_{7} F_{9}\right)\right) \\
\left.\left(D\left(F_{0} F_{3} F_{8} F_{10} F_{11}\right)\right)\right]\end{array}$} \\
\hline
\end{tabular}
target is $31 \%$, rounded to the nearest in teger. Calculation by feature overlap returns the proportion $8 / 12$ for the pair $\mathrm{AB}$. The proportion arises because $\mathrm{A}$ and $\mathrm{B}$ share the four features $F_{0}, F_{2}, F_{3}$, and $F_{6}$, which constitute

Table 1

Empirical Matrix and Feature Definition for a Hypothetical Character Set
8 of the 12 features in the two feature lists for the letters. The pairs $\mathrm{AC}, \mathrm{AD}, \mathrm{BC}, \mathrm{BD}$, and $\mathrm{CD}$ produce the proportions $0,2 / 10,4 / 10,4 / 12$, and $2 / 8$, respectively (i.e., 0 features are shared by $A$ and $C, 2$ of 10 by $A$ and D, 4 of 10 by B and C, 4 of 12 by B and D, and 2 of 8 by $C$ and $D)$. In the example, the values resulting from the computation of feature overlap are the same as those found in the empirical table. The whole target average is $31 \%$.

The calculation of the average for each letter in the target is identical, but only for the relevant pairs. For example, for $A$ we would average the values for $A B$, $\mathrm{AC}$, and $\mathrm{AD}$; for $\mathrm{B}$, the values of $\mathrm{BA}, \mathrm{BC}$, and $\mathrm{BD}$; for $C$, the values of $C A, C B$, and $C D$; and for $D$, the values of $\mathrm{DA}, \mathrm{DB}$, and $\mathrm{DC}$. The individual letter averages would be $32 \%, 47 \%, 22 \%$, and $26 \%$ for A, B, C, and D, respectively, rounded to the nearest integer. Calculation by feature overlap would follow the same procedure as described in the previous paragraph, but only for the relevant pairs.

The calculation of the average for each letter with its adjacent letters is straightforward. Only pairs involving the immediately neighboring letters are involved. For example, using the target $\mathrm{ABCD}$, the average for $\mathrm{A}$ would involve only $A B$, since there is no letter to the left of $A$. The average for $B$ would involve $A B$ and $B C$, the average for $C$ would involve $B C$ and $C D$, and the average for $\mathrm{D}$ would involve $\mathrm{CD}$. Using the hypothetical empirical table, the averages for $\mathrm{A}, \mathrm{B}, \mathrm{C}$, and $\mathrm{D}$ would be $67 \%, 54 \%, 33 \%$, and $25 \%$, respectively, rounded to the nearest integer. The calculation by the feature overlap derivation would be determined as described above, but only for the relevant pairs.

The values in the hypothetical empirical table are the same as those determined using the overlap of hypothetical features. Identity between empirical and computational values is not common. Convergence between these two approaches, or an explanation for the difference between them, is a goal for research.

The output of the program is the same for both derivations but differs depending on the type of average selected. The general format, using examples calculated with the empirical derivation (Gilmore et al., 1979, Table 2) for the whole target selection, are:

$\begin{array}{ll}\text { word }_{1} & \text { DING } \\ \mathrm{s}_{1} & 3 \\ \text { word }_{2} & \text { YORE } \\ \mathrm{s}_{2} & 3 \\ \cdots & \cdots\end{array}$

For the average of an individual letter paired to all others in the target, or for the average involving the adjacent letters, the output is of the form: 


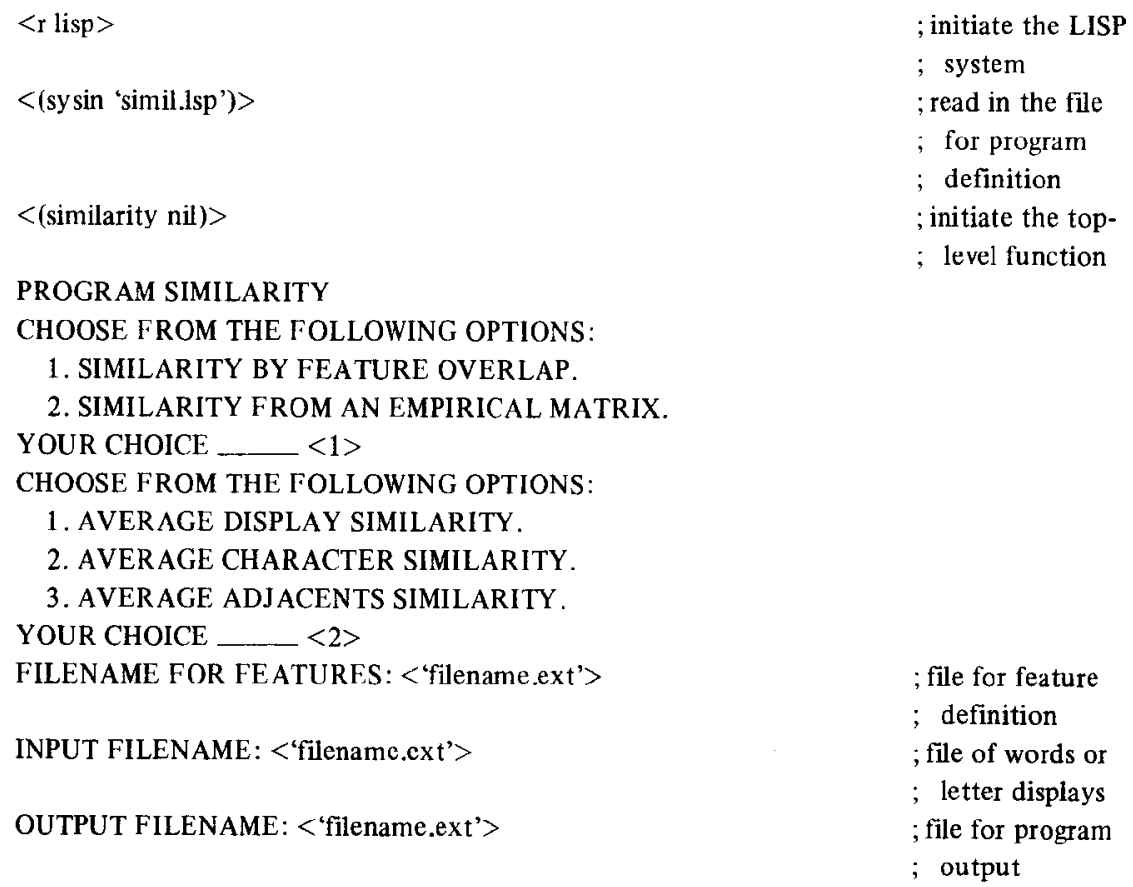

; initiate the LISP

; system

; read in the file

; for program

; definition

; initiate the top-

; level function

Figure 1.

$\begin{array}{cl}\text { word }_{1} & \text { DING } \\ \text { letter }_{1} & \mathrm{D} \\ \mathrm{s}_{1} & 4 \\ \text { letter }_{2} & \mathrm{I} \\ \mathrm{s}_{2} & 1 \\ \ldots & \cdots \\ \text { letter } & \mathrm{G} \\ \mathrm{s}_{\mathrm{n}} & 4 \\ \text { word }_{2} & \text { YORE } \\ \text { letter }_{1} & \text { Y } \\ \mathrm{s}_{1} & 0 \\ \ldots & \cdots\end{array}$

control for similarity relationships in letter displays used in letter recognition research. The program was written as part of an effort to investigate the degree of convergence between empirical and computational approaches. The intimate control in letter displays of variables such as similarity is essential if convergence between empirical and computational approaches is to be attained.

\section{REFERENCES}

Brigos, R., \& Hovecar, D. J. A new distinctive feature theory for upper case letters. Journal of General Psychology, 1975, 93, 87-93.

Geyer, L., \& Dewald, C. Feature lists and confusion matrices Perception \& Psychophysics, 1973, 14, 471-482.

Gilmore, G. C., Hersh, A., Caramazza, A., \& Griffin, J. Multidimensional letter similarity derived from recognition errors. Perception \& Psychophysics, 1979, 25, 425-431.

An example of the program's use is given in Figure 1. In the example, program prompts appear in uppercase and user responses appear in lowercase enclosed in angle brackets. Comments are preceded by a semicolon, which is the comment indicator in DECUS LISP, and are not part of the user dialogue.

The program is provided as an aid to investigate or

Hirata, K., \& Bryden, M. P. Tables of letter sequences varying in order of approximation to English. Psychonomic Science, 1971, 25, 322-324.

KeREN, G., \& BAgaen, S. Recognition models of alphanumeric characters. Perception \& Psychophysics, 1981, 29, $234-246$.

Winston, P., \& Horn, B. LISP. London: Addison-Wesley, 1981.

(Received for publication October 28, 1982; accepted November 27, 1982.) 